\title{
Stat6-Dependent Inhibition of Mincle Expression in Mouse and Human Antigen-Presenting Cells by the Th2 Cytokine IL-4
}

\begin{abstract}
Thomas Hupfer ${ }^{1}$, Judith Schick ${ }^{1}$, Katrin Jozefowski', David Voehringer ${ }^{2}$, Jenny Ostrop ${ }^{1,3 * t}$ and Roland Lang ${ }^{1 * t}$

${ }^{1}$ Institute of Clinical Microbiology, Immunology and Hygiene, Universitätsklinikum Erlangen, Friedrich-Alexander-Universität Erlangen-Nürnberg, Erlangen, Germany, ${ }^{2}$ Department of Infection Biology, Universitätsklinikum Erlangen, Friedrich-Alexander Universität Erlangen-Nürnberg, Erlangen, Germany, ${ }^{3}$ Department of Cancer Research and Molecular Medicine, Centre of Molecular Inflammation Research, Norwegian University of Science and Technology (NTNU), Trondheim, Norway
\end{abstract}

The C-type lectin receptors (CLRs) Mincle, Mcl, and Dectin-2 bind mycobacterial and fungal cell wall glycolipids and carbohydrates. Recently, we described that expression of these CLR is downregulated during differentiation of human monocytes to dendritic cells (DC) in the presence of GM-CSF and IL-4. Here, we demonstrate that the Th2 cytokine IL-4 specifically inhibits expression of Mincle, Mcl, and Dectin-2 in human antigen-presenting cells (APC). This inhibitory effect of IL-4 was observed across species, as murine macrophages and DC treated with IL-4 also downregulated these receptors. IL-4 blocked upregulation of Mincle and Mcl mRNA expression and cell surface protein by murine macrophages in response to the Mincle ligand Trehalose-6,6-dibehenate (TDB), whereas the TLR4 ligand LPS overcame inhibition by IL-4. Functionally, downregulation of Mincle expression by IL-4 was accompanied by reduced cytokine production upon stimulation with TDB. These inhibitory effects of IL-4 were dependent on the transcription factor Stat6. Together, our results show that the key Th2 cytokine IL-4 exerts a negative effect on the expression of Mincle and other Dectin-2 cluster CLR in mouse and human macrophages and DC, which may render these sentinel cells less vigilant for sensing mycobacterial and fungal ligands.

Keywords: C-type lectin receptor, Mincle, Mcl, IL-4, macrophage, monocyte

\section{INTRODUCTION}

Following the identification of Dectin-1 as a Syk-coupled activating receptor for bacterial and fungal beta-glucans 15 years ago (1), C-type lectin receptors (CLRs) have received increasing attention as pattern recognition receptors of the innate immune system. The CLR Dectin-2, Mincle, and Mcl belong to the so-called Dectin-2 family with their genes adjacent to each other in the NK cell receptor gene cluster, localized on human chromosome 12 and mouse chromosome 6 (2).

Abbreviations: CLR, C-type lectin receptor; FcR $\gamma$, Fc receptor gamma chain; IL-4, Interleukin-4; Mincle, macrophageinducible-C-type-lectin; Mcl, macrophage C-type lectin; SYK, spleen tyrosine kinase; TDB, trehalose-6,6-dibehenate; TDM, trehalose-6,6-dimycolate. 
These three receptors recognize mycobacterial and/or fungal ligands, are expressed constitutively or inducibly on innate immune cells, and have an emerging role in innate immunity to these infections.

Mincle (official gene symbol Clec4e, aka Clecsf9) not only binds the mycobacterial cord factor trehalose-6,6-dimycolate (TDM) $(3,4)$, related synthetic glycolipids such as the adjuvant trehalose6,6-dibehenate (TDB) $(3,4)$, but also fungal ligands derived from Candida albicans (5-7), Malassezia furfur $(8,9)$, or Fonsecaea spp. (10, 11). More recently, Mcl (official gene symbol Clec4d, aka Clecsf8) was identified as a second, low affinity-receptor for the mycobacterial cord factor (12), which is required for efficient control of experimental mycobacterial infection in mice (13), but did not bind to sugar ligands on carbohydrate microarrays (14). In contrast, Dectin-2 (gene symbol Clec $4 n$ in mice, CLEC6 $A$ in humans) not only has a classical C-type lectin domain that binds structures with high mannose content from numerous pathogens, most notably Candida albicans $(15,16)$, but also mycobacterial manLAM (17) and schistosomal egg antigen (18).

Whereas Dectin-1 directly recruits the kinase Syk via a nonclassical ITAM motif in its intracellular domain, Mincle, Mcl, and Dectin-2 all associate with the ITAM-containing adapter protein $\mathrm{Fc}$ receptor gamma chain $(\mathrm{FcR} \gamma)$ to initiate signaling through the Card9/Bcl10/Malt1 complex (19). Activation of $\mathrm{NF \kappa B}$ and MAPK pathways triggers substantial reprograming of gene expression in macrophages after activation of Mincle by TDB, similar to Curdlan-induced Dectin-1 activation, but only partially overlapping with inflammatory gene expression induced by TLR ligands (20). Similar to Curdlan, activation of APC by TDB or TDM directs a cytokine milieu fostering the development of Th17 responses to co-delivered protein antigens via production of IL-6, IL-23, and IL-1 (4, 20-24).

Expression of Mincle is strongly inducible in murine macrophages and DC by PAMPs, such as the TLR4 ligand LPS (25) or by its ligand TDM itself $(4,12)$, and depends on the transcription factor $\operatorname{C} / \operatorname{EBP} \beta(25,26)$. Interestingly, Mincle expression is constitutively high in murine monocytes and granulocytes $(21,27)$, similar to human monocytes and macrophages (28). In contrast, expression of $\mathrm{Mcl}$ is constitutively higher in mouse bone marrow-derived macrophages (BMM) and bone marrowderived dendritic cells (BMDC), and inducible to a lesser extent $(12,26)$. Dectin-2 expression is predominantly myeloid restricted and upregulated by inflammatory stimuli (16). Cytokines involved in the upregulation of Dectin-2 include TNF or GM-CSF (29).

IL- 4 is the prototypical Th2 cytokine and induces alternative macrophage activation through the transcription factor Stat6 (30). Interestingly, Th2 responses and IL-4 driven alternative macrophage activation have been associated with poorer outcomes in fungal (31) and in mycobacterial infection $(32,33)$. It is well established that IL-4 induces the expression of Dectin-1 (34). Its effects on the expression of other CLRs are not well characterized, although downregulation of Dectin-2 expression in IL-4 treated human CD14 ${ }^{+}$monocytes has been described (29).

We recently observed a strong downregulation of the mRNA expression of Mincle, $\mathrm{Mcl}$, and Dectin-2 during differentiation of human DC from CD14 ${ }^{+}$monocytes in the presence of GM-CSF and IL-4 in vitro (28). Here, we investigated the regulation of expression of these CLRs by IL-4 in human and mouse APC. Our data show that IL-4 specifically downregulates Mincle, Mcl, and Dectin-2 expression, but not Dectin-1 expression, in both species and impairs Mincle-dependent macrophage activation in response to the cord factor analog TDB.

\section{MATERIALS AND METHODS}

\section{Isolation and Culture of Human Antigen-Presenting Cells}

The use of human leukocytes from healthy donors with written informed consent complies with the Declaration of Helsinki (Ethical committee Erlangen approval no. 4055 and no. 111_12 B). PMBCs were obtained from leukoreduction system chambers by density centrifugation (35). Monocytes were positively selected from PBMC using $\alpha$-CD14 microbeads (Miltenyi Biotec), purity was $\geq 90 \%$. For culture, RPMI1640 was supplemented with $10 \%$ (v/v) fetal calf serum (FCS, Biochrom) and $100 \mathrm{U} / \mathrm{ml}$ penicillin and $100 \mu \mathrm{g} / \mathrm{ml}$ streptomycin (cRPMI). A total of $50 \mathrm{U} / \mathrm{ml} \mathrm{GM-CSF}$ (Genzyme) or $50 \mathrm{U} / \mathrm{ml}$ M-CSF (Peprotech) were added for differentiation of macrophages. $50 \mathrm{U} / \mathrm{ml} \mathrm{GM-CSF}$ and $250 \mathrm{U} / \mathrm{ml}$ IL-4 (Peprotech) were added for differentiation of DC. Cells were cultured at a density of $0.8 \times 10^{6}$ cells $/ \mathrm{ml}$ (GM-CSF macrophages, DC) or $1.6 \times 10^{6}$ cells $/ \mathrm{ml}$ (M-CSF macrophages) for 6-7 days without change of media at $37^{\circ} \mathrm{C}$ with $5 \% \mathrm{CO}_{2}$ humidified air.

\section{Isolation and Culture of Mouse Macrophages and DC}

$\mathrm{C} 57 \mathrm{BL} / 6, \mathrm{Clec} \mathrm{e}^{-/-}, \mathrm{Balb} / \mathrm{c}$, and Stat6 ${ }^{-/-}$mice were bred at the Präklinische Experimentelle Tierzentrum of the Medical Faculty of the Friedrich-Alexander-University Erlangen-Nürnberg. Mice were housed and humanely sacrificed according to regional government regulations. Bone marrow cells from femurs and tibiae were differentiated to macrophages by culture in complete Dulbecco's Modified Eagle Medium containing 10\% FCS, antibiotics, and $50 \mu \mathrm{M} \beta$-mercaptoethanol (cDMEM) plus 10\% L929cell conditioned medium as a source of M-CSF, as previously described (36). On day 7, adherent macrophages were harvested by Accutase (Sigma) treatment, washed and counted. For generation of BMDC, bone marrow cells were cultured in CDMEM containing $10 \%$ conditioned medium of X63 cells producing GM-CSF for 7-8 days before harvesting. Peritoneal exudate cells (PEC) were obtained on day 4 after injection of mice with $2 \mathrm{ml}$ of $4 \%$ thioglycollate by flushing the peritoneal cavity with $10 \mathrm{ml}$ of ice-cold PBS.

\section{Cell Stimulation}

Human antigen-presenting cells were stimulated by adding M-CSF (Peprotech, $50 \mathrm{U} / \mathrm{ml}$ ), GM-CSF (Genzyme, $50 \mathrm{U} / \mathrm{ml}$ ), or IL-4 (Peprotech, $250 \mathrm{U} / \mathrm{ml}$ unless otherwise stated) to the cell culture medium before plating of cells into 48 well cell-culture plates in a concentration of $0.85 \times 10^{6} / \mathrm{ml}$. Mouse antigenpresenting cells were stimulated with $10 \mathrm{ng} / \mathrm{ml} \mathrm{IL-4}$ (Peprotech), $10 \mathrm{ng} / \mathrm{ml}$ LPS (E. coli serotype O55:B5, Sigma) by adding the substance to the cell culture medium before plating of the cells into 24 well cell-culture plates in a concentration of $0.5 \times 10^{6} / \mathrm{ml}$. 
TDB (Polar Avanti, $5 \mu \mathrm{g} / \mathrm{ml}$ ) was used plate-bound as previously described $(4,37)$, coating with isopropanol only was used as a negative control.

\section{mRNA Expression Analysis of CLRs}

RNA was isolated with Trifast (Peqlab), cDNA was transcribed using a cDNA synthesis kit (Applied Biosystems). Expression levels of the housekeeping genes hypoxanthine-guaninphosphoribosyltransferase (Hprt, mouse) or cyclophilin A (PPIA, human), as of the genes of interest, were analyzed using primer/probe combinations selected from the Roche Universal Probe Library (Roche). For human MINCLE, the primer/probe combination was selected using the software PrimerExpress (Applied Biosystems). All primers and probes used have been described (28) and were purchased from Metabion. $\Delta$ CT values were calculated as $\Delta \mathrm{CT}=\mathrm{CT}_{\text {(housekeeping gene) }}-\mathrm{CT}_{\text {(gene of interest) }}$ (such that higher values indicate higher relative expression), and $\Delta \Delta \mathrm{CT}$ values referred to the calibrator as indicated.

\section{Cytokine ELISA}

Cytokine concentration of murine G-CSF and TNF- $\alpha$ was analyzed by sandwich ELISA (DuoSet ELISA, R\&D Systems) using cell-free cell culture supernatants of cells stimulated as indicated.

\section{Flow Cytometry of Mincle and Mcl Surface Receptor Levels}

The $2 \times 10^{5} \mathrm{BMM}$ of C57BL/ 6 and Mincle-/- mice were stimulated with LPS $(10 \mathrm{ng} / \mathrm{ml})$ or TDB $(5 \mu \mathrm{g} / \mathrm{ml})$ in the presence or absence of IL-4 (10 ng/ml), as well as with IL-4 alone, for $16 \mathrm{~h}$ in F-bottom 96 well cell-culture plates. Cell surface expression of Mincle and Mcl was analyzed by flow cytometry. Staining was performed by using anti-Mincle (clone 4A9, MBL) and anti-Mcl (clone 3A4) (38) as primary antibodies, and anti-rat IgG1 conjugated to APC as secondary antibody (eBioscience) in a final concentration of $1 \mu \mathrm{g} / \mathrm{ml}$ for anti-Mincle and anti-rat IgG-APC or $2.27 \mu \mathrm{g} / \mathrm{ml}$ for anti-Mcl. Fc receptors were blocked by adding anti-mouse CD16/32 (clone 93, eBioscience) in a final concentration of $2.5 \mu \mathrm{g} / \mathrm{ml}$ before staining. Cells were stained with primary antibodies for $20 \mathrm{~min}$ at $4^{\circ} \mathrm{C}$, washed, and then stained with secondary antibody for $20 \mathrm{~min}$ at $4^{\circ} \mathrm{C}$. Flow cytometry data were acquired on a FACSCanto II instrument (BD); analysis was carried out using FlowJo (version 10).

\section{Statistics}

Statistical analysis was performed using Prism5 (GraphPad Software, v5.01). Student's $t$-test was applied as indicated for non-paired and paired testing between two groups. ${ }^{\star} p<0.05$, ${ }^{* *} p<0.01,{ }^{* *} p<0.001$, ns $p>0.05$.

\section{RESULTS}

\section{IL-4 Downregulates MINCLE Expression in Human Monocyte-Derived APC}

In our recent characterization of CLR expression on primary human antigen-presenting cells, we observed higher expression of MINCLE mRNA in macrophages generated with M-CSF or GM-CSF compared to DC differentiated in the presence of GM-CSF plus IL-4 (28). This effect was confirmed in independent experiments using cells from unrelated healthy donors, showing a strong reduction in the $\Delta \mathrm{CT}$ values from around -4.05 (M-CSF) and -5.0 (GM-CSF) to -10.4 (GM-CSF + IL-4) (Figure 1A). Quantitative RT-PCR analysis of mRNA expression for a range of CLR showed that the reduced expression in DC compared to GM-CSF-derived macrophages was selectively observed for MINCLE, MCL, DECTIN2, and CLEC12A whereas expression levels of the other tested CLR were not significantly different (Figure 1B). Since the presence of IL-4 in the cell culture media was the main difference in the differentiation protocols for GM-CSF macrophages and DC, we tested whether IL-4 alone would be sufficient to downregulate MINCLE expression in monocyte cultures (Figure 1C). MINCLE expression was highest in freshly isolated CD14 ${ }^{+}$ monocytes and declined over time during culture in media alone. However, addition of IL-4 caused a stronger reduction, and this effect was detectable at all time points tested (6-144 h). The presence of GM-CSF kept MINCLE expression at a high level, but additional IL-4 induced strong downregulation, which was again detectable already after $6 \mathrm{~h}$, but increased over time (Figure 1C). The inhibitory effect of IL-4 on MINCLE expression by monocytes was dose dependent and already detected with $0.25 \mathrm{U} / \mathrm{ml} \mathrm{IL}-4$ but more pronounced at concentrations above $2.5 \mathrm{U} / \mathrm{ml}$ (Figure 1D). Having shown that IL-4 inhibits MINCLE expression in monocytes early during differentiation cultures, we next asked whether the same effect was seen when adding IL-4 to macrophages or DC after differentiation. While the expression of MINCLE in monocyte-derived DC cultured in media only was relatively variable and the reduction observed for the average values in DC treated with IL-4 was not significant, both types of macrophages responded with significant downregulation of MINCLE when treated with IL-4 for $48 \mathrm{~h}$ (Figure 1E).

\section{IL-4 also Downregulates Mincle Expression in Murine Antigen-Presenting Cells}

We next asked whether the regulatory action of IL-4 is conserved between species and tested the expression of mouse macrophages and DC treated for $48 \mathrm{~h}$ with IL-4 (Figure 2). IL-4 was used at the saturating concentration of $10 \mathrm{ng} / \mathrm{ml}$ (39). Mincle mRNA levels in M-CSF-driven BMM under basal conditions were lower than those in $\mathrm{d} 4$ thioglycollate-elicited peritoneal exsudate cells (PEC) or GM-CSF-driven BMDC, but IL-4 decreased Mincle expression in all three cell types significantly (Figure 2A). Mcl expression was comparably high in untreated BMM, PEC, and BMDC and strongly inhibited by IL-4 (Figure 2B). Dectin-2 mRNA was highest in BMDC and significantly downregulated by IL-4 in BMM and BMDC (Figure 2C). In contrast, IL-4 did not inhibit Dectin-1 expression in mouse APC, but in fact increased Dectin-1 mRNA in BMM, confirming a previous report (34) (Figure 2D). Together, the pattern of IL-4 effects on expression of the CLR family members examined was very similar between 

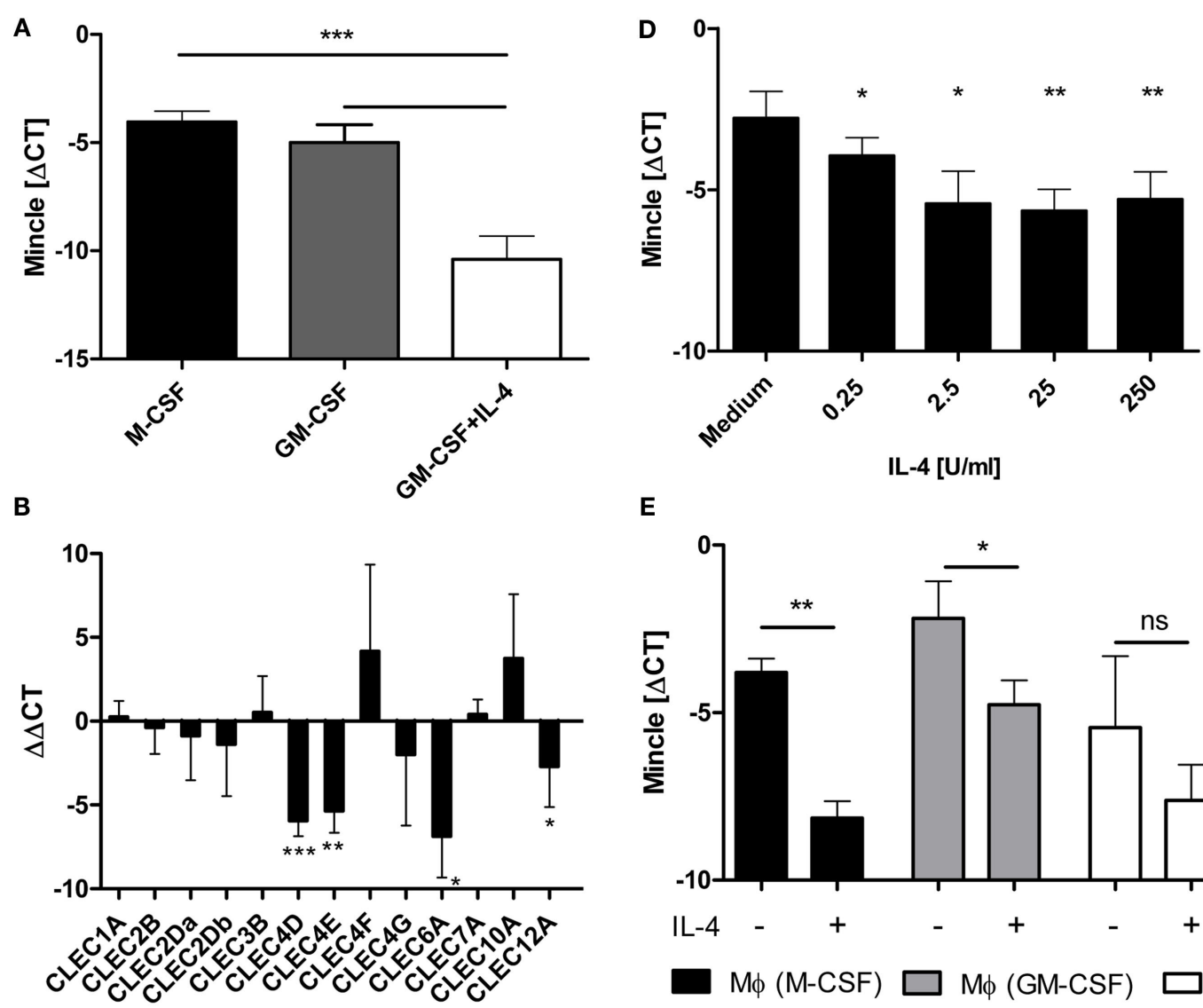

E
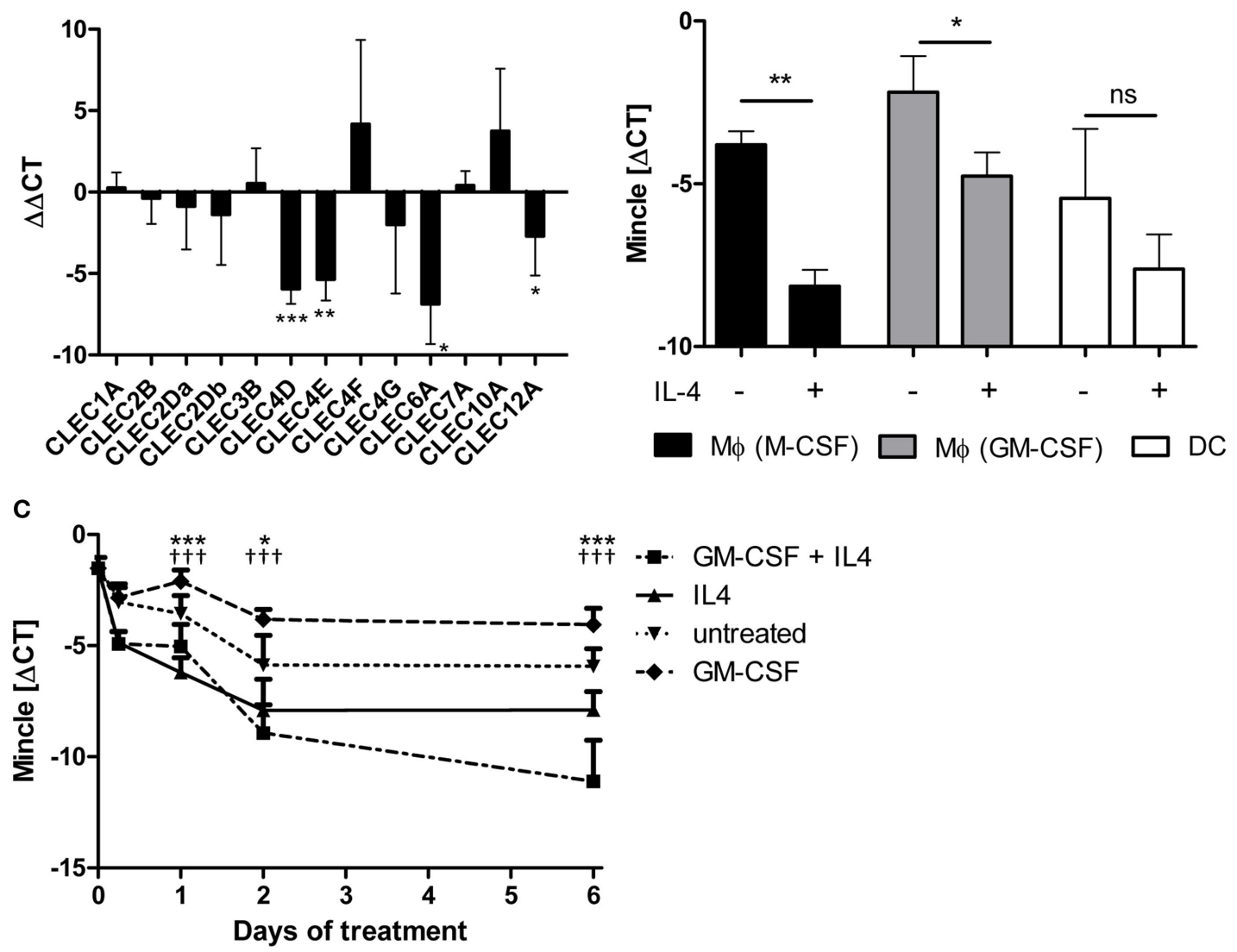

FIGURE 1 | IL-4 downregulates Mincle mRNA expression in human antigen-presenting cells. (A) Monocyte-derived DC express less MINCLE than monocyte-derived macrophages. Human CD14+ cells were differentiated for 6 days in the presence of M-CSF or GM-CSF (to obtain macrophages) or GM$\mathrm{CSF}+\mathrm{IL}-4$ (to obtain dendritic cells). After that, MINCLE mRNA levels were measured using qPCR. The data are depicted as mean \pm SD of $\triangle \mathrm{CT}$ values $\left(\mathrm{Ct}_{[\mathrm{PPIA}]}-\mathrm{Ct}_{[\mathrm{MINCLE}}\right)$ of cells from eight independent blood donors. (B) Reduced expression in DC is restricted to MINCLE, MCL, DECTIN2, and CLEC12A. Gene expression of human GM-CSF-elicited macrophages was compared to dendritic cells (induced via GM-CSF + IL-4) after 6 days of differentiation using qPCR. The data are depicted as mean \pm SD of $\Delta \Delta$ CT values (using GM-CSF M $\phi$ as calibrators) from 6 independent blood donors. (C) IL-4 is sufficient to reduce MINCLE expression in human CD14+ cells. CD14+ peripheral blood mononuclear cells were stimulated as indicated. MINCLE expression was determined using qPCR. The data are depicted as mean $\pm \mathrm{SD}(n=2$ for $\mathrm{d} 0, n=4$ for $\mathrm{d} 0.25$, and $n=6$ for all other time points). Asterisks indicate significant $p$-values for the comparison "IL-4 vs. untreated", crosses for the comparison "GM-CSF + IL-4 vs. GM-CSF". (D) Dose-dependence of IL-4 effect. CD14+ peripheral blood mononuclear cells were stimulated with increasing concentrations of IL-4 for $24 \mathrm{~h}$. Mean \pm SD of $\Delta C T$ values for MINCLE expression of cells from four independent donors. (E) Human CD14+ monocyte-derived M-CSF or GM-CSF elicited macrophages and dendritic cells were stimulated with IL-4 or left untreated for 48 h. Subsequently, MINCLE expression was determined using qPCR. The data are depicted as mean \pm SD of cells from four independent blood donors. Statistical significance was tested using a paired $t$-test. ${ }^{*} p<0.05,{ }^{* *} p<0.01,{ }^{* *} p<0.001,{ }^{+t+} p<0.001$. 

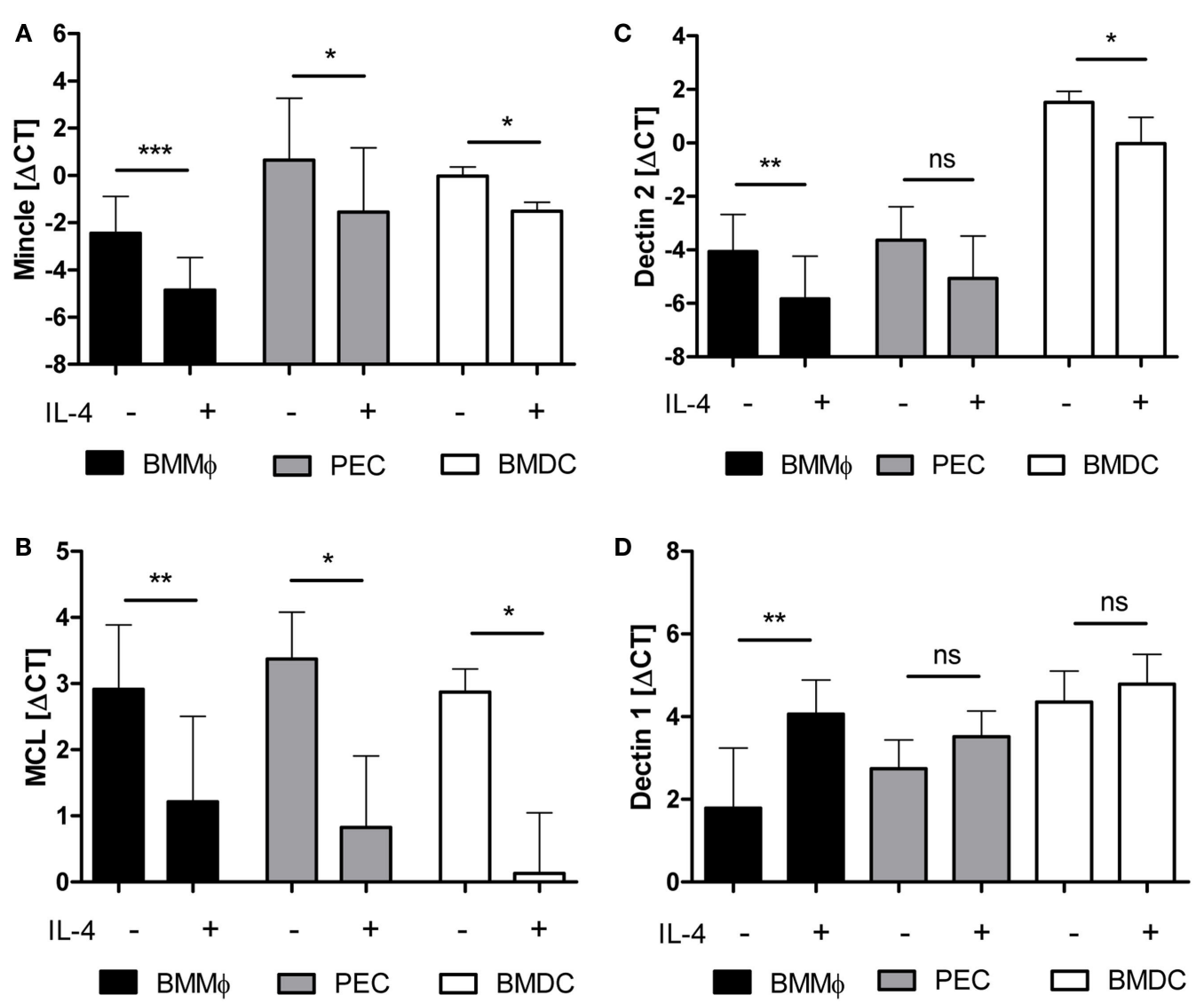

FIGURE 2 | mRNA expression of Mincle, Mcl, and Dectin-2 is downregulated by IL-4 on murine antigen-presenting cells. Murine bone marrow-derived macrophages (BMM, black bars), thioglycolate-elicited peritoneal macrophages (PEC, gray bars), or bone marrow-derived dendritic cells (BMDC, white bars) treated or not with IL-4 for $48 \mathrm{~h}$ were analyzed by qRT-PCR for expression of mRNA encoding Mincle (A), Mcl (B), Dectin-2 (C), or Dectin-1 (D). Mean \pm SD of $\Delta$ CT values from eight (BMM) or three (PEC and BMDC) independent experiments. Statistical significance was tested using a paired $t$-test. ${ }^{*} p<0.05,{ }^{* *} p<0.01,{ }^{\star * *} p<0.001$.

mouse and human, with significant downregulation of Mincle, $\mathrm{Mcl}$, and Dectin-2, whereas Dectin-1 was not affected or even upregulated by IL-4.

\section{Differential Regulation of TDB- or LPS-Inducible Mincle Expression by IL-4}

Expression of Mincle is strongly inducible by pattern recognition receptor signaling, such as TLR or CLR $(4,25,40)$. Therefore, we examined the effect of IL-4 on Mincle mRNA expression in BMM treated with the TLR4 ligand LPS or the Mincle ligand TDB (Figure 3). Stimulation with LPS or TDB for $48 \mathrm{~h}$ significantly induced Mincle expression, as expected; the concomitant exposure to IL-4 inhibited Mincle upregulation by TDB, but not by LPS (Figure 3A). Priming of BMM with LPS or LPS + IL-4 overnight, followed by removal of LPS and washing before addition of IL-4, also abrogated the inhibitory effect of IL- 4 on Mincle expression (Figure 3B). Thus, the inhibitory effect of IL-4 on Mincle expression could be overcome by stimulation of macrophages with the TLR4 ligand LPS.

\section{IL-4 Abrogates Cell Surface Expression of Mincle and Mcl Protein in Response to TDB but Not LPS}

We next employed flow cytometry to determine the cell surface levels of Mincle and $\mathrm{Mcl}$ in resting and stimulated macrophages (Figures 4A,B). Confirming previous reports $(40,41)$, only weak staining with the Mincle- and Mcl-specific monoclonal antibodies (4A9 and 3A4) was detected in resting macrophages. In fact, using Mincle-deficient macrophages as staining controls, only a minimal increase in the fluorescence signal for Mincle was observed. Overnight stimulation with TDB caused upregulation of Mincle and $\mathrm{Mcl}$ on a significant fraction of the macrophage population. However, this increase was almost completely prevented by co-treatment with IL-4. In contrast, the LPS-induced surface staining for Mincle and Mcl was found on the entire macrophage population and was only weakly reduced in the presence of IL-4. Together, the changes in cell surface protein levels and mRNA expression of Mincle and $\mathrm{Mcl}$ showed a close correlation. 



FIGURE 3 | LPS overcomes IL-4-induced downregulation of Mincle mRNA expression. (A) Murine bone marrow-derived macrophages were treated with TDB or LPS, with or without IL-4, or were left untreated for $48 \mathrm{~h}$. (B) LPS-priming abrogates the effect of IL-4 on Mincle expression. Bone marrow-derived murine macrophages were primed overnight with LPS (P: LPS) or left untreated (P: -). The next day, LPS was washed off, the cells were re-plated and cultivated for $48 \mathrm{~h}$ with or without IL-4. Mincle expression was determined with qPCR. The data are depicted as mean \pm SD of $\Delta$ CT values from five independent experiments. Statistical significance was tested using a paired $t$-test. ${ }^{* *} p<0.01$

\section{IL-4 Inhibits G-CSF and TNF Expression and Secretion by Macrophages in Response to TDB}

We reasoned that inhibition of Mincle expression in macrophages treated with IL-4 may functionally impair the response to the Mincle ligand TDB. To test this hypothesis, we determined the levels of the cytokines G-CSF and TNF, which are robustly secreted by macrophages stimulated with TDB or with LPS although the levels varied considerably between independent experiments (Figures 5A,B). Macrophages treated with IL-4 or media alone did not produce significant amounts of G-CSF or TNF (data not shown). Concomitant treatment with IL-4 strongly and consistently inhibited the production of both cytokines in response to TDB (Figures $\mathbf{5 A}, \mathbf{B}$ ). In contrast, LPSinduced G-CSF was inhibited less strongly by IL-4 (Figure 5A), and the LPS-induced release of TNF was not affected by IL-4 (Figure 5B).

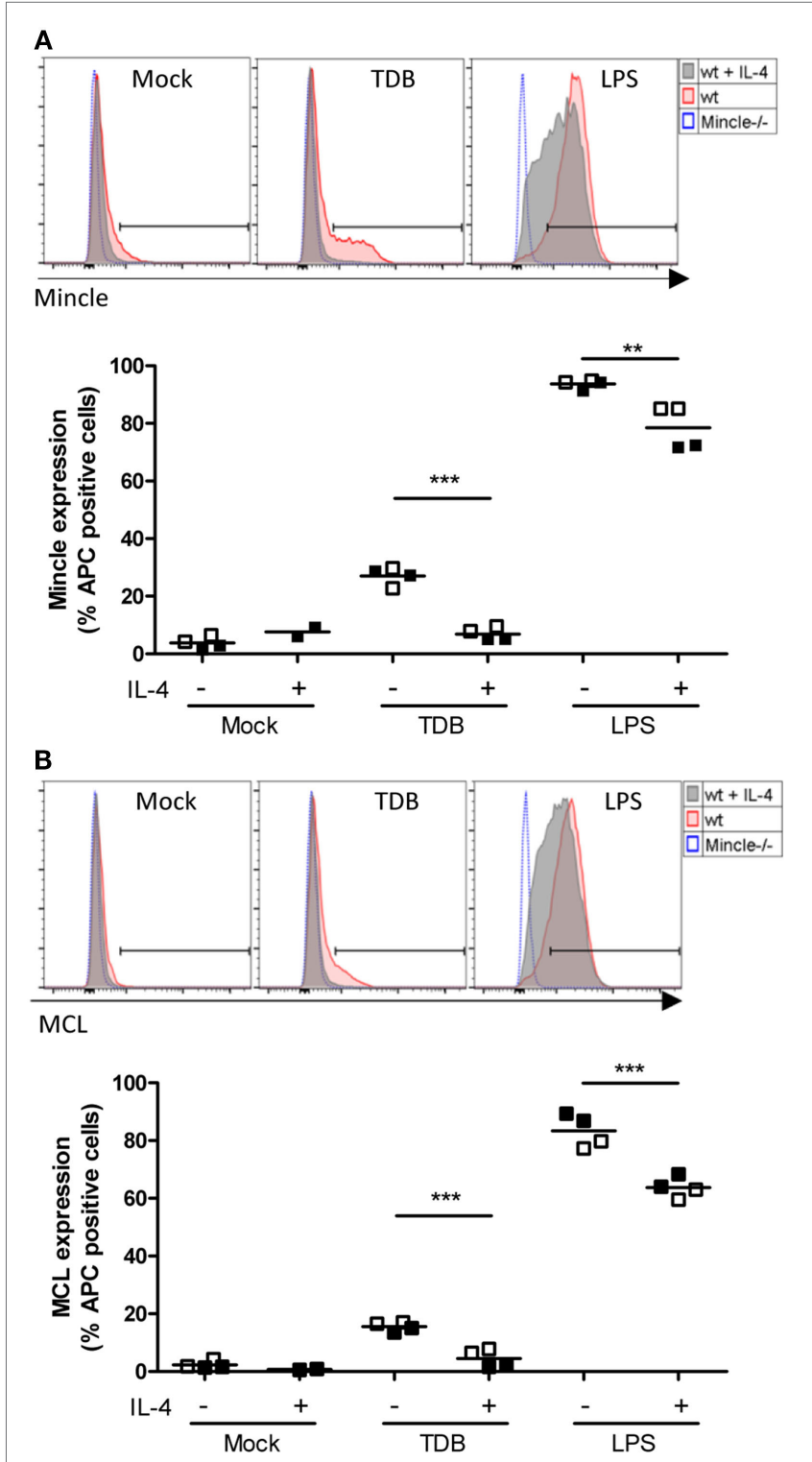

FIGURE 4 | Regulation of cell surface levels of Mincle and $\mathrm{Mcl}$ in murine macrophages by IL-4. Bone marrow-derived macrophages were analyzed for Mincle (A) and $\mathbf{M c l}$ (B) surface expression $16 \mathrm{~h}$ after stimulation as indicated by using anti-Mincle (4A9) or anti-Mcl (3A4) primary antibodies followed by staining with APC-conjugated secondary antibody. (A) Histograms show Mincle surface expression in C57BL/6 BMMs (wt) stimulated with TDB or LPS or left untreated (Mock) in presence (gray filled) or absence (red filled) of IL-4 as indicated. Mincle-/BMMs (blue dotted) were stimulated with TDB or LPS or left untreated and used as negative control. Histograms are from one representative experiment. Scatter plot shows Mincle expression depicted as percentage of APC-positive cells from C57BL/6 BMMs after stimulation with TDB or LPS in presence or absence of IL-4 or left untreated (Mock). Data are depicted from two independent experiments performed in duplicates (filled symbols: exp. 1, open symbols: exp. 2). (B) Histograms and scatter plot show Mcl surface expression, respectively. Cells were treated as described in (A). Statistical significance was tested using an unpaired $t$-test. ${ }^{\star \star} p<0.01,{ }^{\star \star \star} p<0.001$. 

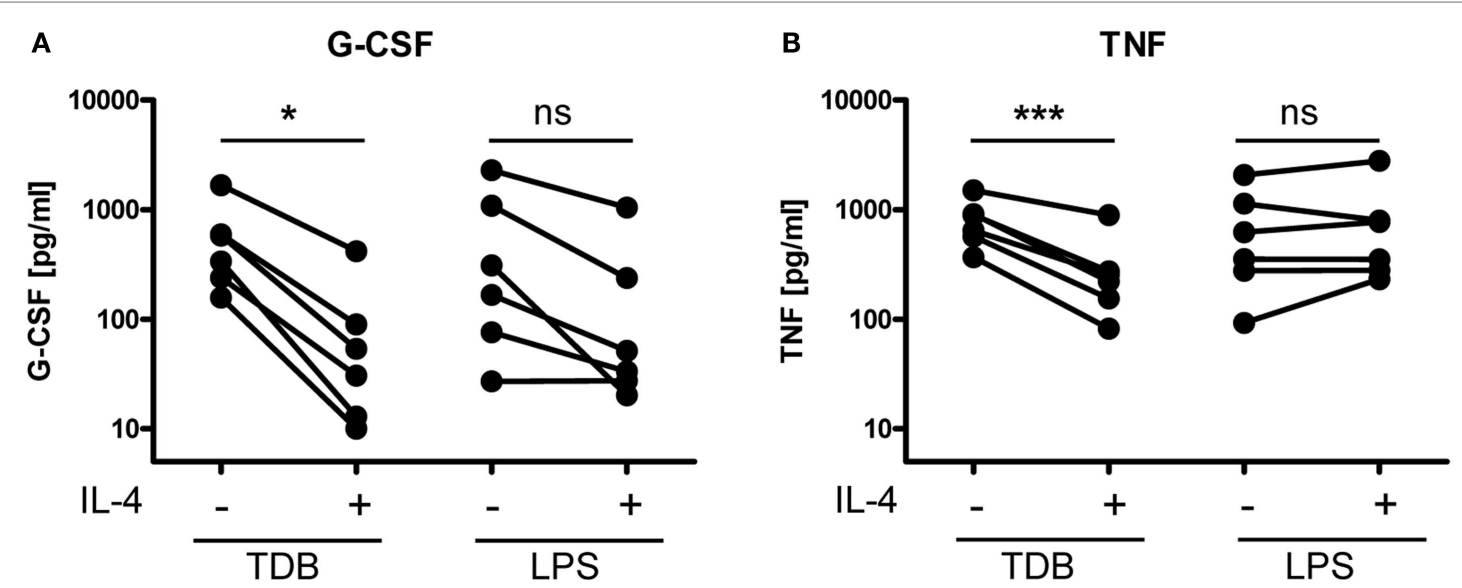

FIGURE 5 | IL-4 inhibits TDB-induced cytokine production in murine macrophages. Bone marrow-derived macrophages were stimulated for 24 h with TDB or LPS in the presence or absence of IL-4. Cytokine levels in cell culture supernatant were quantified using ELISA [(A), G-CSF, and (B), TNF]. (A,B) Depicted are mean ELISA values of replicates from six independent experiments. Supernatants of BMM treated with media or IL-4 alone did not contain significant amounts of G-CSF or TNF (data not shown). Statistical significance of the effect of IL-4 on stimulation with TDB and LPS, respectively, was tested using a two-sided paired $t$-test. ${ }^{*} p<0.05,{ }^{* *} p<0.001$.

\section{Negative Regulation of Mincle Expression and Target Gene Expression by IL-4 Requires Stat6}

IL-4 triggers Jak-Stat signaling, with Stat6 being the pivotal transcription factor for IL-4-induced gene expression (42). We investigated whether the inhibitory effect of IL-4 was also dependent on this transcription factor by using Stat $6^{-/-}$BMM in comparison to the respective Balb/c wild type control BMM (Figure 6). The Stat6 genotype had no effect on the basal (data not shown) or TDB-induced Mincle expression (Figure 6A). However, IL-4 inhibited the basal (data not shown) and TDB-induced Mincle expression in Balb/c BMM, but not in Stat $6^{-1-}$ BMM (Figure 6A). Similarly, the TDB-inducible expression of G-CSF at the mRNA and protein level was strongly inhibited in Balb/c BMM, but not in Stat6 $^{-1-}$ BMM, where in fact a moderate increase in G-CSF protein secretion production was evident (Figures 6B,C).

\section{DISCUSSION}

Expression of pattern recognition receptors is a prerequisite for sensitive detection of microbial pathogens and is often restricted to specialized cell types of the innate immune system. While the CLR Mincle, Dectin-2, and $\mathrm{Mcl}$ are all expressed in myeloid cells, their expression levels are differentially affected by microbial and inflammatory stimuli. In this manuscript, we show that the prototypical Th2 cytokine IL-4 downregulates expression of these members of the Dectin-2 family, but not of other CLR, e.g., Dectin-1. Importantly, this inhibitory effect of IL-4 was conserved across species, as it was detected in human as well as murine APC. Reduced receptor expression may be functionally relevant, because production of cytokines in response to the Mincle ligand TDB was reduced in IL-4-treated macrophages. Our findings raise several questions concerning the mechanism and potential consequences of Dectin-2 family regulation by IL- 4 during immune responses.

Signaling by the IL-4 receptor activates the transcription factor Stat6, which is required for most transcriptional responses (42). Therefore, the dependence of negative regulation of Mincle expression on Stat6 was not surprising. Further investigation will be required to determine in detail how Stat6 mediates directly or indirectly the decrease in Mincle mRNA expression. One possibility is that Stat6 interferes with the recruitment of activating transcription factors to the promoter/ enhancer regions of Mincle, $\mathrm{Mcl}$, and Dectin-2. The transcription factor $\mathrm{C} / \mathrm{EBP} \beta$ is essential for the inducible expression of Mincle, as shown already by Matsumoto et al. in 1999 (25), but also for upregulation of Dectin-2 and $\mathrm{Mcl}$ in response to TDB, whereas it is not required for Dectin-1 expression (26). Thus, the inhibitory effect of IL- 4 and the requirement for C/EBP $\beta$ in inducible receptor expression correlate. It is conceivable that IL-4 may downregulate C/EBP $\beta$ at the transcriptional or post-transcriptional level in macrophages. Stat6 and C/EBP $\beta$ cooperate in the induction of several genes associated with Th2 responses (43) and alternative macrophage activation, such as Arginase-1 $(44,45)$. However, to our knowledge, no inhibition of $\mathrm{C} / \mathrm{EBP} \beta$-dependent transcriptional responses by Stat6 has been described to date. To answer these questions, chromatin immunoprecipitation assays for direct binding of $\mathrm{C} / \mathrm{EBP} \beta$ and Stat 6 to the promoter regions of Mincle, $\mathrm{Mcl}$, and Dectin-2 will be required in future experiments.

Although IL-4 potently inhibited Mincle expression during differentiation of DC from human monocytes or in mouse macrophages, strikingly, the concurrent stimulation or priming of macrophages with the TLR4 ligand LPS overcame the IL-4 effect and prevented Mincle downregulation (Figure 3). It is possible that LPS interferes with IL-4 signaling through induction of negative regulators, such as Socs $1(46,47)$. However, LPS did not 

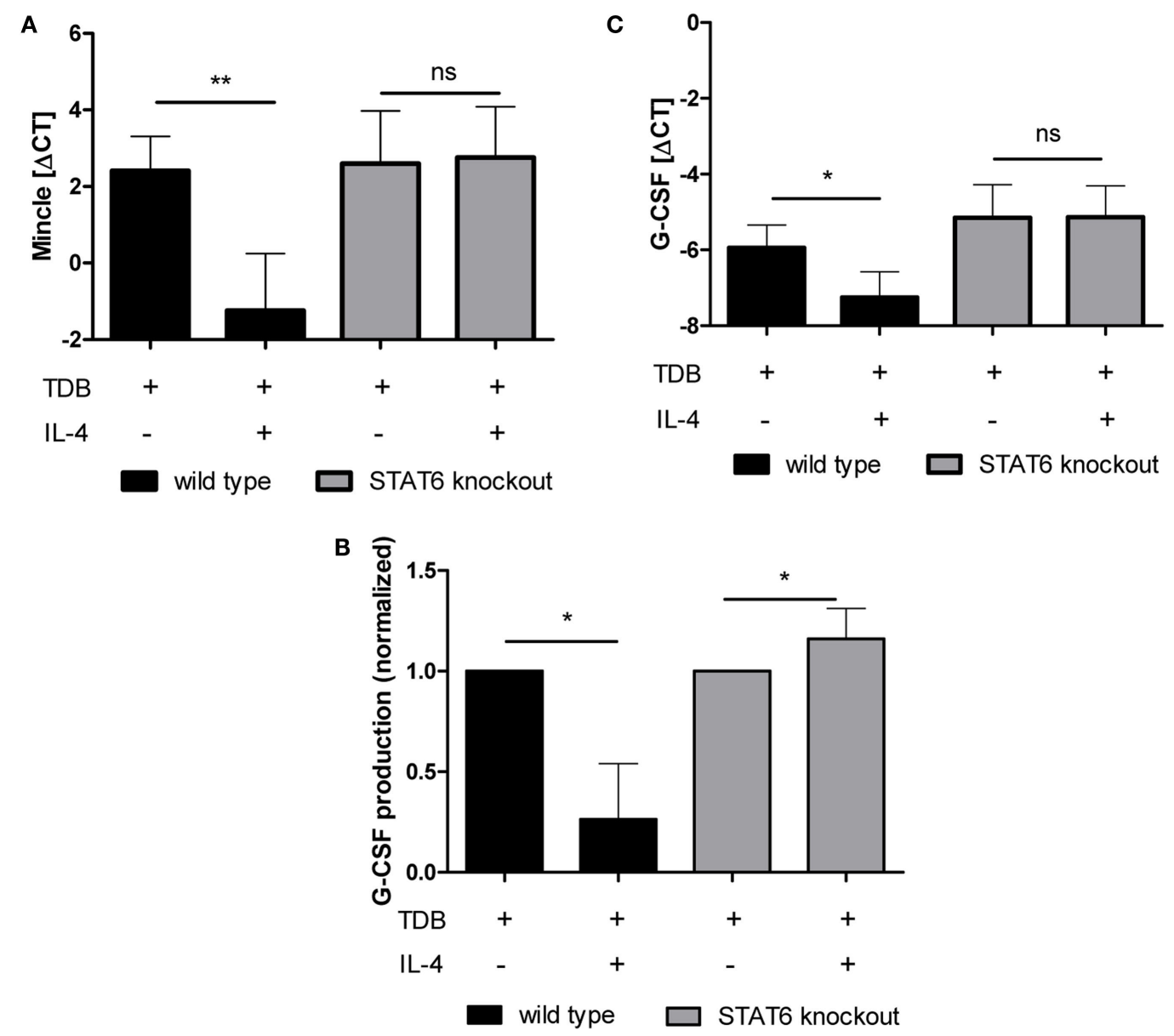

FIGURE 6 | IL-4-mediated inhibition of TDB-induced Mincle expression and G-CSF production is STAT6-dependent. Bone marrow-derived macrophages from STAT6-deficient mice and Balb/c wild-type controls were stimulated for $48 \mathrm{~h}$ with TDB in the presence or absence of IL-4. Mincle and G-CSF expression was determined by qPCR $(\mathbf{A}, \mathbf{C})$, and G-CSF concentration in the cell supernatant was obtained using ELISA (B). ELISA values were normalized to TDB-induced G-CSF production. Mean \pm SD of four independent experiments. Statistical significance was tested using a paired $t$-test. ${ }^{\star} p<0.05,{ }^{\star \star} p<0.01$.

block the induction of IL-4 induced Arginase-1 expression but rather synergistically induced it (data not shown), suggesting that the abrogation of the inhibitory IL-4 effect on Mincle expression likely involves other mechanisms. Regardless of the mechanism, it appears that TLR ligands make macrophages unresponsive to IL-4-triggered downregulation of Mincle expression. This effect would be consistent with the notion that the presence of TLR ligands signals the requirement for continued high level expression of CLR, regardless of other signals which otherwise would reduce their expression.

We have also shown here that macrophages treated with IL-4 not only express less Mincle but also fail to respond to TDB stimulation with upregulation of Mincle and $\mathrm{Mcl}$ (Figures 3 and 4), and of the cytokines G-CSF and TNF (Figure 5), indicating that reduced receptor expression may lead to weaker macrophage activation in response to mycobacterial cord factor. It remains to be tested in the future whether IL-4-induced downregulation of Mincle, $\mathrm{Mcl}$, and Dectin-2 functionally impairs innate recognition of and the response to mycobacteria and fungi. From a technical point of view, our results also suggest that the use of growth factors and cytokines in protocols for generation of DC and macrophages, i.e. inclusion of IL-4 or not, may have strong effects on the response to CLR ligands. In the case of mycobacteria, the combined recognition of TDM by Mincle and $\mathrm{Mcl}$, and of manLAM by Dectin-2, would be affected by IL-4, which may lead to compromised macrophage responsiveness to mycobacteria. It is interesting to speculate that in Th2-primed individuals reduced expression of Mincle, $\mathrm{Mcl}$, and Dectin-2 on myeloid cells may occur, which could impair the detection of mycobacteria, and thereby could contribute to increased susceptibility to mycobacterial infection. Coinfection with helminths and M. tuberculosis is prevalent in many parts of the world. Epidemiological data show that exposure of household contacts to patients with active pulmonary TB resulted in higher risk to develop latent tuberculosis in the case of concurrent helminth infection, consistent with an impaired capacity of the innate immune cells, in this case alveolar macrophages, to kill mycobacteria upon inhalation (48). 
In addition, anti-helminthic treatment of people latently infected with $M$. tuberculosis led to marked improvement of $\mathrm{T}$ cell responses, which could also be at least partially due to upregulation of CLR expression with loss of helminth-induced IL-4 $(49,50)$. Indeed, such a mechanism appears to operate for levels of TLR2 and TLR9 (49). In the mouse model of coinfection, experimental evidence for increased susceptibility of helminth-infected animals challenged with M. tuberculosis has been obtained for increased mycobacterial burden for coinfection with Schistosoma mansoni or Nippostrongylus brasiliensis $(32,51)$. While helminth-induced IL-4 probably acts at multiple levels on the innate as well as the development of adaptive immune responses (52), the possible contribution of impaired expression of Mincle, $\mathrm{Mcl}$, and Dectin-2 to modulation of the host response in tuberculosis in individuals co-infected with worms should therefore be explored in future studies.

\section{REFERENCES}

1. Brown GD, Gordon S. Immune recognition. A new receptor for beta-glucans. Nature (2001) 413:36-7. doi:10.1038/35099612

2. Kerscher B, Willment JA, Brown GD. The dectin-2 family of c-type lectin-like receptors: an update. Int Immunol (2013) 25:271-7. doi:10.1093/intimm/ dxt006

3. Ishikawa E, Ishikawa T, Morita YS, Toyonaga K, Yamada H, Takeuchi O, et al. Direct recognition of the mycobacterial glycolipid, trehalose dimycolate, by c-type lectin mincle. J Exp Med (2009) 206:2879-88. doi:10.1084/ jem. 20091750

4. Schoenen H, Bodendorfer B, Hitchens K, Manzanero S, Werninghaus K, Nimmerjahn F, et al. Cutting edge: Mincle is essential for recognition and adjuvanticity of the mycobacterial cord factor and its synthetic analog trehalose-dibehenate. J Immunol (2010) 184:2756-60. doi:10.4049/ jimmunol.0904013

5. Bugarcic A, Hitchens K, Beckhouse AG, Wells CA, Ashman RB, Blanchard H. Human and mouse macrophage-inducible C-type lectin (Mincle) bind Candida albicans. Glycobiology (2008) 18:679-85. doi:10.1093/glycob/cwn046

6. Vijayan D, Radford KJ, Beckhouse AG, Ashman RB, Wells CA. Mincle polarizes human monocyte and neutrophil responses to Candida albicans. Immunol Cell Biol (2012) 90:889-95. doi:10.1038/icb.2012.24

7. Wells CA, Salvage-Jones JA, Li X, Hitchens K, Butcher S, Murray RZ, et al. The macrophage-inducible c-type lectin, mincle, is an essential component of the innate immune response to Candida albicans. J Immunol (2008) 180:7404-13. doi:10.4049/jimmunol.180.11.7404

8. Ishikawa T, Itoh F, Yoshida S, Saijo S, Matsuzawa T, Gonoi T, et al. Identification of distinct ligands for the C-type lectin receptors mincle and dectin-2 in the pathogenic fungus malassezia. Cell Host Microbe (2013) 13:477-88. doi:10.1016/j.chom.2013.03.008

9. Yamasaki S, Matsumoto M, Takeuchi O, Matsuzawa T, Ishikawa E, Sakuma M, et al. C-type lectin mincle is an activating receptor for pathogenic fungus, malassezia. Proc Natl Acad Sci U S A (2009) 106:1897-902. doi:10.1073/ pnas.0805177106

10. Sousa Mda G, Reid DM, Schweighoffer E, Tybulewicz V, Ruland J, Langhorne J, et al. Restoration of pattern recognition receptor costimulation to treat chromoblastomycosis, a chronic fungal infection of the skin. Cell Host Microbe (2011) 9:436-43. doi:10.1016/j.chom.2011.04.005

11. Wevers BA, Kaptein TM, Zijlstra-Willems EM, Theelen B, Boekhout T, Geijtenbeek TB, et al. Fungal engagement of the C-type lectin mincle suppresses dectin-1-induced antifungal immunity. Cell Host Microbe (2014) 15:494-505. doi:10.1016/j.chom.2014.03.008

12. Miyake Y, Toyonaga K, Mori D, Kakuta S, Hoshino Y, Oyamada A, et al. C-type lectin $\mathrm{mcl}$ is an fcrgamma-coupled receptor that mediates the adjuvanticity of mycobacterial cord factor. Immunity (2013) 38:1050-62. doi:10.1016/ j.immuni.2013.03.010

\section{AUTHOR CONTRIBUTIONS}

$\mathrm{TH}$, JS, and JO performed experiments and analyzed data. KJ performed experiments. DV provided critical reagents. JO and $R L$ designed experiments and wrote the manuscript.

\section{ACKNOWLEDGMENTS}

The present work was performed in (partial) fulfillment of the requirements for obtaining the degree "Dr. med." of TH. Support by the Deutsche Forschungsgemeinschaft to the lab of RL is gratefully acknowledged (SFB 796, TP B6; GRK 1660, TP A2). TH was supported by a doctoral fellowship provided by the IZKF of the Medical Faculty at the Friedrich-Alexander-University ErlangenNürnberg. We thank Dr. Janet Willment for the generous gift of anti-Mcl antibody.

13. Wilson GJ, Marakalala MJ, Hoving JC, van Laarhoven A, Drummond RA, Kerscher B, et al. The C-type lectin receptor Clecsf8/Clec4d is a key component of anti-mycobacterial immunity. Cell Host Microbe (2015) 17:252-9. doi:10.1016/j.chom.2015.01.004

14. Graham LM, Gupta V, Schafer G, Reid DM, Kimberg M, Dennehy KM, et al. The C-type lectin receptor CLECSF8 (CLEC4D) is expressed by myeloid cells and triggers cellular activation through Syk kinase. J Biol Chem (2012) 287:25964-74. doi:10.1074/jbc.M112.384164

15. Robinson MJ, Osorio F, Rosas M, Freitas RP, Schweighoffer E, Gross O, et al. Dectin-2 is a Syk-coupled pattern recognition receptor crucial for th17 responses to fungal infection. J Exp Med (2009) 206:2037-51. doi:10.1084/ jem.20082818

16. Taylor PR, Reid DM, Heinsbroek SE, Brown GD, Gordon S, Wong SY. Dectin-2 is predominantly myeloid restricted and exhibits unique activation-dependent expression on maturing inflammatory monocytes elicited in vivo. Eur J Immunol (2005) 35:2163-74. doi:10.1002/eji.200425785

17. Yonekawa A, Saijo S, Hoshino Y, Miyake Y, Ishikawa E, Suzukawa M, et al. Dectin-2 is a direct receptor for mannose-capped lipoarabinomannan of mycobacteria. Immunity (2014) 41:402-13. doi:10.1016/j.immuni.2014.08.005

18. Ritter M, Gross O, Kays S, Ruland J, Nimmerjahn F, Saijo S, et al. Schistosoma mansoni triggers dectin-2, which activates the nlrp3 inflammasome and alters adaptive immune responses. Proc Natl Acad Sci U S A (2010) 107:20459-64. doi:10.1073/pnas.1010337107

19. Sancho D, Reis e Sousa C. : Signaling by myeloid C-type lectin receptors in immunity and homeostasis. Annu Rev Immunol (2012) 30:491-529. doi:10.1146/annurev-immunol-031210-101352

20. Werninghaus K, Babiak A, Gross O, Holscher C, Dietrich H, Agger EM, et al. Adjuvanticity of a synthetic cord factor analogue for subunit mycobacterium tuberculosis vaccination requires fcrgamma-Syk-Card9-dependent innate immune activation. J Exp Med (2009) 206:89-97. doi:10.1084/jem.20081445

21. Desel C, Werninghaus K, Ritter M, Jozefowski K, Wenzel J, Russkamp N, et al. The mincle-activating adjuvant TDB induces Myd88-dependent Th1 and Th17 responses through IL-1R signaling. PLoS One (2013) 8:e53531. doi:10.1371/journal.pone.0053531

22. LeibundGut-Landmann S, Gross O, Robinson MJ, Osorio F, Slack EC, Tsoni SV, et al. Syk- and CARD9-dependent coupling of innate immunity to the induction of thelper cells that produce interleukin 17. Nat Immunol (2007) 8:630-8. doi:10.1038/ni1460

23. Shenderov K, Barber DL, Mayer-Barber KD, Gurcha SS, Jankovic D, Feng CG, et al. Cord factor and peptidoglycan recapitulate the Th17-promoting adjuvant activity of mycobacteria through mincle/CARD9 signaling and the inflammasome. J Immunol (2013) 190:5722-30. doi:10.4049/jimmunol.1203343

24. Schweneker K, Gorka O, Schweneker M, Poeck H, Tschopp J, Peschel C, et al. The mycobacterial cord factor adjuvant analogue trehalose-6,6'-dibehenate (TDB) activates the nlrp3 inflammasome. Immunobiology (2013) 218:664-73. doi:10.1016/j.imbio.2012.07.029 
25. Matsumoto M, Tanaka T, Kaisho T, Sanjo H, Copeland NG, Gilbert DJ, et al. A novel lps-inducible C-type lectin is a transcriptional target of NF-IL6 in macrophages. J Immunol (1999) 163:5039-48.

26. Schoenen H, Huber A, Sonda N, Zimmermann S, Jantsch J, Lepenies B, et al. Differential control of mincle-dependent cord factor recognition and macrophage responses by the transcription factors C/Ebpbeta and Hiflalpha. J Immunol (2014) 193:3664-75. doi:10.4049/jimmunol.1301593

27. Lee WB, Kang JS, Yan JJ, Lee MS, Jeon BY, Cho SN, et al. Neutrophils promote mycobacterial trehalose dimycolate-induced lung inflammation via the mincle pathway. PLoS Pathog (2012) 8:e1002614. doi:10.1371/journal.ppat. 1002614

28. Ostrop J, Jozefowski K, Zimmermann S, Hofmann K, Strasser E, Lepenies B, et al. Contribution of mincle-Syk signaling to activation of primary human APCS by mycobacterial cord factor and the novel adjuvant TDB. J Immunol (2015) 195:2417-28. doi:10.4049/jimmunol.1500102

29. Gavino AC, Chung JS, Sato K, Ariizumi K, Cruz PD Jr. Identification and expression profiling of a human C-type lectin, structurally homologous to mouse dectin-2. Exp Dermatol (2005) 14:281-8. doi:10.1111/j.0906-6705.2005. 00312.x

30. Van Dyken SJ, Locksley RM. Interleukin-4- and interleukin-13-mediated alternatively activated macrophages: roles in homeostasis and disease. Annu Rev Immunol (2013) 31:317-43. doi:10.1146/annurev-immunol-032712-095906

31. Romani L. Immunity to fungal infections. Nat Rev Immunol (2011) 11:275-88. doi:10.1038/nri2939

32. Potian JA, Rafi W, Bhatt K, McBride A, Gause WC, Salgame P. Preexisting helminth infection induces inhibition of innate pulmonary anti-tuberculosis defense by engaging the IL-4 receptor pathway. J Exp Med (2011) 208:1863-74. doi:10.1084/jem.20091473

33. Salgame P, Yap GS, Gause WC. Effect of helminth-induced immunity on infections with microbial pathogens. Nat Immunol (2013) 14:1118-26. doi:10.1038/ ni. 2736

34. Willment JA, Lin HH, Reid DM, Taylor PR, Williams DL, Wong SY, et al. Dectin-1 expression and function are enhanced on alternatively activated and GM-CSF-treated macrophages and are negatively regulated by IL-10, dexamethasone, and lipopolysaccharide. J Immunol (2003) 171:4569-73. doi:10.4049/jimmunol.171.11.6297-b

35. Pfeiffer IA, Zinser E, Strasser E, Stein MF, Dorrie J, Schaft N, et al. Leukoreduction system chambers are an efficient, valid, and economic source of functional monocyte-derived dendritic cells and lymphocytes. Immunobiology (2013) 218:1392-401. doi:10.1016/j.imbio.2013.07.005

36. Hammer M, Mages J, Dietrich H, Schmitz F, Striebel F, Murray PJ, et al. Control of dual-specificity phosphatase-1 expression in activated macrophages by IL-10. Eur J Immunol (2005) 35:2991-3001. doi:10.1002/eji.200526192

37. Ozeki Y, Tsutsui H, Kawada N, Suzuki H, Kataoka M, Kodama T, et al. Macrophage scavenger receptor down-regulates mycobacterial cord factor-induced proinflammatory cytokine production by alveolar and hepatic macrophages. Microb Pathog (2006) 40:171-6. doi:10.1016/ j.micpath.2005.12.006

38. Kerscher B, Wilson GJ, Reid DM, Mori D, Taylor JA, Besra GS, et al. Mycobacterial receptor, Clec4d (Clecsf8, MCL), is coregulated with mincle and upregulated on mouse myeloid cells following microbial challenge. Eur J Immunol (2016) 46:381-9. doi:10.1002/eji.201545858

39. Schleicher U, Paduch K, Debus A, Obermeyer S, Konig T, Kling JC, et al. TNF-mediated restriction of arginase 1 expression in myeloid cells triggers type 2 NO synthase activity at the site of infection. Cell Rep (2016) 15:1062-75. doi:10.1016/j.celrep.2016.04.001

40. Kerscher B, Dambuza IM, Christofi M, Reid DM, Yamasaki S, Willment JA, et al. Signalling through myd88 drives surface expression of the mycobacterial receptors MCL (Clecsf8, Clec4d) and mincle (Clec4e) following microbial stimulation. Microbes Infect (2016) 18(7-8):505-9. doi:10.1016/ j.micinf.2016.03.007

41. Miyake Y, Oh-Hora M, Yamasaki S. C-type lectin receptor MCL facilitates mincle expression and signaling through complex formation. Jimmunol (2015) 194:5366-74. doi:10.4049/jimmunol.1402429

42. Goenka S, Kaplan MH. Transcriptional regulation by stat6. Immunol Res (2011) 50:87-96. doi:10.1007/s12026-011-8205-2

43. Mikita T, Kurama M, Schindler U. Synergistic activation of the germline epsilon promoter mediated by stat6 and c/ebp beta. J Immunol (1998) 161:1822-8.

44. Gray MJ, Poljakovic M, Kepka-Lenhart D, Morris SM Jr. Induction of arginase I transcription by IL-4 requires a composite DNA response element for STAT6 and C/EBPbeta. Gene (2005) 353:98-106. doi:10.1016/j.gene.2005.04.004

45. Pauleau AL, Rutschman R, Lang R, Pernis A, Watowich SS, Murray PJ. Enhancer-mediated control of macrophage-specific arginase I expression. J Immunol (2004) 172:7565-73. doi:10.4049/jimmunol.172.12.7565

46. Dickensheets HL, Venkataraman C, Schindler U, Donnelly RP. Interferons inhibit activation of stat 6 by interleukin 4 in human monocytes by inducing SOCS-1 gene expression. Proc Natl Acad Sci U S A (1999) 96:10800-5. doi:10.1073/pnas.96.19.10800

47. Losman JA, Chen XP, Hilton D, Rothman P. Cutting edge: SOCS-1 is a potent inhibitor of IL-4 signal transduction. J Immunol (1999) 162:3770-4.

48. Verhagen LM, Hermans PW, Warris A, de Groot R, Maes M, Villalba JA, et al. Helminths and skewed cytokine profiles increase tuberculin skin test positivity in warao amerindians. Tuberculosis (Edinb) (2012) 92:505-12. doi:10.1016/j.tube.2012.07.004

49. Babu S, Bhat SQ, Kumar NP, Anuradha R, Kumaran P, Gopi PG, et al. Attenuation of toll-like receptor expression and function in latent tuberculosis by coexistent filarial infection with restoration following antifilarial chemotherapy. PLoS Negl Trop Dis (2009) 3:e489. doi:10.1371/journal.pntd. 0000489

50. Elias D, Wolday D, Akuffo H, Petros B, Bronner U, Britton S. Effect of deworming on human $t$ cell responses to mycobacterial antigens in helminthexposed individuals before and after Bacille Calmette-Guerin (BCG) vaccination. Clin Exp Immunol (2001) 123:219-25. doi:10.1046/j.1365-2249. 2001.01446.x

51. Frantz FG, Rosada RS, Peres-Buzalaf C, Perusso FR, Rodrigues V, Ramos SG, et al. Helminth coinfection does not affect therapeutic effect of a DNA vaccine in mice harboring tuberculosis. PLoS Negl Trop Dis (2010) 4:e700. doi:10.1371/ journal.pntd.0000700

52. George PJ, Anuradha R, Kumar NP, Sridhar R, Banurekha VV, Nutman TB, et al. Helminth infections coincident with active pulmonary tuberculosis inhibit mono- and multifunctional $\mathrm{Cd} 4+$ and $\mathrm{Cd} 8+\mathrm{T}$ cell responses in a process dependent on IL-10. PLoS Pathog (2014) 10:e1004375. doi:10.1371/ journal.ppat.1004375

Conflict of Interest Statement: The authors declare that the research was conducted in the absence of any commercial or financial relationships that could be construed as a potential conflict of interest.

Copyright (c) 2016 Hupfer, Schick, Jozefowski, Voehringer, Ostrop and Lang. This is an open-access article distributed under the terms of the Creative Commons Attribution License (CC BY). The use, distribution or reproduction in other forums is permitted, provided the original author(s) or licensor are credited and that the original publication in this journal is cited, in accordance with accepted academic practice. No use, distribution or reproduction is permitted which does not comply with these terms. 